\title{
Experiences of Nurses in the Process of Determining a Nursing Diagnosis and Needs for Applying a Nursing Diagnosis: Fostering Understanding to Support the Use of Nursing Diagnoses in Clinical Practice
}

\author{
Yasuko Kume", Harumi Yamaguchi
}

Graduate School of Nursing, School of Nursing, Mukogawa Women's University Hyogo, Japan

\author{
Article Details \\ Article Type: Research Article \\ Received date: $06^{\text {th }}$ January, 2017 \\ Accepted date: $27^{\text {th }}$ March, 2017 \\ Published date: $31^{\text {st }}$ March, 2017
}

"Corresponding Author: Yasuko Kume, Graduate School of Nursing, School of Nursing, Mukogawa Women's University, Hyogo, Japan. E-mail: kume@mukogawa-u.ac.jp

Citation: Kume Y, Yamaguchi H (2017) Experiences of Nurses in the Process of Determining a Nursing Diagnosis and Needs for Applying a Nursing Diagnosis: Fostering Understanding to Support the Use of Nursing Diagnoses in Clinical Practice. J Comp Nurs Res Care 2: 107. https://doi.org/10.33790/jenrc1100107.

Copyright: $(\mathbb{2} 2017$, This is an open-access article distributed under the terms of the Creative Commons Attribution License 4.0, which permits unrestricted use, distribution, and reproduction in any medium, provided the original author and source are credited.

\begin{abstract}
Purpose: This study illustrates through experiences of nurses while determining nursing diagnoses in terms of thought, behavior, and emotion. It demonstrates current issues in and the needs for support in the use of nursing diagnoses.
\end{abstract}

Methods: Data were collected through conducting semi-structured interviews based on an interview guide concerning the experiences of nurses and their awareness of issues in the process of determining nursing diagnoses.

Principal Results and Major Conclusions: Followingten categories were selected upon considering the experiences of nurses in determining nursing diagnoses from a thought perspective: [Comparison with defining characteristics], [Confirmation of defining characteristics, related factors, and definition], [Searching for potential nursing diagnoses from main symptoms and problems], [Considering nursing diagnosis from nursing intervention and outcome], [Patterned thinking relating to specific diagnoses], [Considering some temporary nursing diagnoses and monitoring progress], [Identification of multiple nursing diagnoses], [Comparison of some nursing diagnosesby pickingpotential diagnoses of defining characteristics or related factors, definition], [Integrating multiple nursing diagnoses], and [Avoiding nursing diagnosis decisions]. From the behavioral and emotional perspectives, emphasis was placed on [Confirming and reviewing with other staff to determine a diagnosis] and [Solo confirmation and review to decide on a nursing diagnosis]. These were found to be driven by: [Pressure to provide a nursing diagnosis], [Sense of burden over task load that goes with nursing diagnoses], [Anxiety when determining a nursing diagnosis], [Negative emotions towards nursing diagnoses], and [Decline of negative emotions towards nursing diagnoses]. Needs for nursing diagnosis application included [Needs for fostering understanding and learning of nursing diagnosis], [Needs for expanding the process of nursing diagnosis], [Needs for nursing diagnosis and nursing intervention for patients in specific situations], [Needs for the application of an electronic health record system], and [Needs for thinking ability in applying nursing diagnosis], suggesting a need to foster understanding of nursing diagnoses and application of examples.

\section{Introduction}

According to the Nursing Diagnosis Association International (NANDA-I), "A nursing diagnosis is a clinical judgment concerning a human response to health conditions/life processes, or a vulnerability for that response, by an individual, family, group, or community. A nursing diagnosis provides the basis for selection of nursing interventions to achieve outcomes for which the nurse has accountability" (p. 464) [1]. It is also an important tool for keeping records of one's thought process and practice when practicing nursing.

Seventy-eight percent of office-based physicians used some form of an electronic health record (EHR) system in 2013 [2]. It is reported that the introduction rate for electronic health record systems in Japan is $27.3 \%$, with over $70.1 \%$ of hospitals having more than 400 beds [3]. The prevalence of nursing diagnoses and use of an electronic medical recoding system might have the same tendency, indicating that nursing diagnosis is primarily being spread to medical facilities with a large number of beds. Consequently, there have also been studies conducted regarding the difficulties and issues with using nursing diagnoses, highlighting the fact that psychiatric nursing units have few nursing diagnostic labels, insufficient understanding of nursing diagnostic definitions and concepts, and insufficient assessment [4]. Issues concerning the accuracy of diagnoses have also been pointed out in a study of nursing diagnostic ability using mock cases [5]. As issues are still expected to arise in the application of nursing diagnosis, we considered it necessary to investigate what nurses experience in the process of determining nursing diagnoses, what is lacking in order to apply nursing diagnosis in practice, what difficulties exist, and what needs exist.

\section{Purpose}

This study explored the experiences of nurses in the process of determining nursing diagnoses in terms of thought, behavior, and emotion. It also examined the needs for the use of nursing diagnoses and considered current issues and a support system for making improvements based on these needs.

Methods of Data Collection and Analysis

Since this study focuses on interpreting the experiences of nurses 
by focusing on learning and reflection based on experience from the characteristics of nursing practices, it explored these experiences from the three perspectives of thought, behavior, and emotion under the Action and Looking back on the action and Awareness of essential aspects and Creating alternative methods of action, and Trial (ALACT) model proposed by Korthagen [6]. The meanings of thoughts, behaviors, and emotions were explored in the following manner. The thought perspective signifies what nurses were thinking during the process of determining a nursing diagnosis. The behavioral perspective signifies what actions the nurses actually took during this process. Finally, the emotional perspective signifies what the nurses felt during this process. In addition, needs required for applying nursing diagnosis refers to that which is recognized as lacking and as being difficult in the process of determining nursing diagnoses, as well as that which needs to be fulfilled.

Participating this study were 13 female nurses who work at Hospital A and agreed to participate in this study. Five of these nurses work in the internal medicine ward, two of them work in the surgical ward, and six of them work in the mixed medical ward. Hospital A is a general hospital with 499 beds in the Kansai region of Japan. Years of clinical experience among the participants varied from two years to 18 years with an average of $9.2 \pm 5.8$ years. Data were collected by conducting semi-structured interviews based on an interview guide concerning the experiences of nurses and their awareness of issues in the process of determining nursing diagnoses. Data analysis was performed using a content analysis method [7], and records of the interviews were prepared. The whole of the transcript from the nurses' testimony was considered for context, and details of their testimony were noted. Individual details of the notes were summarized and expressions indicating important details within that text were sampled along with tendencies in thought, behavior, emotion, and awareness of problems. Categorization was also done based on similarities and integrated into both categories and subcategories. The reliability of the analysis was also verified between three researchers to ensure the process of analysis remained faithful to the data contents. Collection of data was conducted from March to September of 2015.

\section{Ethical Considerations}

The primary aim of the study, the voluntary nature of participation, and strict observance of anonymity were explained to the participants of the study, and interviews were conducted once consent was given. A private room was prepared for conducting interviews, and interviews were generally done once within 30 minutes to an hour time and location were adjusted according to the interviewee's convenience. Approval was obtained from the Ethics Committee of Mukogawa Women's University and from the institution that conducted the interviews.

\section{Results}

\section{Experiences of nurses in the process for determining nursing diagnosis.}

Thought perspective of nurses in the process of determining a nursing diagnosis (refer to Table 1).

\begin{tabular}{|c|c|}
\hline Category & Subcategory \\
\hline \multirow[t]{2}{*}{ 1. Comparison with defining characteristics } & $\begin{array}{l}\text { 1. Deciding on nursing diagnosis that conforms more to } \\
\text { diagnostic indicators }\end{array}$ \\
\hline & $\begin{array}{l}\text { 2. Differentiating between some diagnoses from less match } \\
\text { defining characteristics }\end{array}$ \\
\hline \multirow[t]{3}{*}{$\begin{array}{l}\text { 2. Confirmation of defining characteristics, related fac- tors, and } \\
\text { definition }\end{array}$} & $\begin{array}{l}\text { 3. Considering multiple possible nursing diagnoses that } \\
\text { partially match the definition and defining charac- teristics, } \\
\text { and related factors }\end{array}$ \\
\hline & $\begin{array}{l}\text { 4. Determining priority nursing diagnoses that match the } \\
\text { definition and defining characteristics, and related factors }\end{array}$ \\
\hline & $\begin{array}{l}\text { 5. Determining priority nursing diagnoses that better match the } \\
\text { definition and defining characteristics, and related factors }\end{array}$ \\
\hline \multirow[t]{2}{*}{$\begin{array}{l}\text { 3. Searching for potential nursing diagnoses from main symptoms } \\
\text { and problems }\end{array}$} & $\begin{array}{l}\text { 6. Searching for potential nursing diagnoses that are considered } \\
\text { from problems }\end{array}$ \\
\hline & $\begin{array}{l}\text { 7. Searching for potential nursing diagnoses with em- phasis } \\
\text { on symptoms and complaints }\end{array}$ \\
\hline \multirow[t]{2}{*}{$\begin{array}{l}\text { 4. Considering nursing diagnosis from nursing inter- ventions and } \\
\text { outcomes }\end{array}$} & $\begin{array}{l}\text { 8. Consideration of nursing diagnosis based on the ne- cessity } \\
\text { of nursing intervention }\end{array}$ \\
\hline & $\begin{array}{l}\text { 9. Consideration for the relationship between nursing } \\
\text { diagnoses and outcome }\end{array}$ \\
\hline \multirow[t]{3}{*}{ 5. Patterned thinking relating to specific diagnoses } & 10. Nursing diagnoses linked with specific patient conditions \\
\hline & 11. Nursing diagnoses linked with past experiences \\
\hline & $\begin{array}{l}\text { 12. Nursing diagnoses linked with defining character- istics } \\
\text { and related factors }\end{array}$ \\
\hline \multirow{2}{*}{$\begin{array}{l}\text { 6. Considering some temporary nursing diagnoses and monitoring } \\
\text { progress }\end{array}$} & 13. Referencing nursing diagnoses while lacking infor- mation \\
\hline & 14. Referencing nursing diagnoses when unsure \\
\hline
\end{tabular}

Table. 1 Cont......... 


\begin{tabular}{|c|c|}
\hline \multirow[t]{5}{*}{ 7. Identification of multiple nursing diagnoses } & 15. Identification based on condition and restrictions \\
\hline & $\begin{array}{l}\text { 16. Identification from priority of nursing intervention based } \\
\text { on patient's condition }\end{array}$ \\
\hline & 17. Identification based on definition of nursing diag- noses \\
\hline & $\begin{array}{l}\text { 18. Identification by confirming the number of con- forming } \\
\text { defining characteristics and related factors }\end{array}$ \\
\hline & $\begin{array}{l}\text { 19. Comparison of defining characteristics and defini- tions for } \\
\text { nursing diagnosis approximated from prob- lems }\end{array}$ \\
\hline \multirow{2}{*}{$\begin{array}{l}\text { 8. Comparison of some nursing diagnoses by picking potential } \\
\text { diagnoses of defining characteristics or relat- ed factors, definition }\end{array}$} & 20. Consideration of some unfamiliar nursing diagnoses \\
\hline & 21. Searching some impossible nursing diagnoses \\
\hline \multirow[t]{2}{*}{ 9. Integrating multiple nursing diagnoses } & $\begin{array}{l}\text { 22. Integrating to reduce burden and confusion due to } \\
\text { overlapping nursing intervention and evaluation }\end{array}$ \\
\hline & 23. Integration to design high priority nursing diagno- ses \\
\hline \multirow[t]{2}{*}{ 10. Avoiding determining some nursing diagnoses } & 24. Avoiding determining impossible nursing diagnoses \\
\hline & 25. Avoiding determining unfamiliar nursing diagnoses \\
\hline
\end{tabular}

Table 1 Thought perspective of nurses in the process of determining nursing diagnosis

Ten categories were selected from the thought perspective of nurses in the process of determining nursing diagnoses. They include [Comparison with defining characteristics], [Confirmation of defining characteristics, related factors, and definition], [Searching for potential nursing diagnoses from main symptoms and problems], [Considering nursing diagnosis from nursing interventions and outcomes], [Patterned thinking relating to specific diagnoses], [Considering some temporary nursing diagnoses and monitoring progress], [Identification of multiple nursing diagnoses], [Comparison of some nursing diagnoses by picking potential diagnoses of defining characteristics or related factors, definition],[Integrating multiple nursing diagnoses], and [Avoiding determining some nursing diagnoses These consist of a further 25 sub-categories.

[Comparison with defining characteristics] consists of two sub-categories, including $<$ Deciding on nursing diagnosis that conforms more to diagnostic indicators>, which stems from one nurse's response that "It is some what difficult to understand the defining characteristics, so I simplify things, come up with my own understanding of what is going on, and look at the figures to see if they conform with the diagnostic indicators and related indicators."

[Searching for potential nursing diagnoses from main symptoms and problems] consists of the subcategory $<$ Searching for nursing diagnoses that are considered from problems $>$, which came from responses such as "You discover problems such as distress, look at diagnoses to see what it falls under, and find a label that seems to fit only to look at the details and see it's not a match. It basically boils down to searching for diagnosis labels related to problems by looking for anything that seems similar." It also includes the subcategory $<$ Searching for potential nursing diagnoses with emphasis on symptoms and complaints $>$, which is based on the response that "sometimes the patient will have an extreme complaint or distress, so you think there must be a nursing diagnosis and search for one."

[Considering nursing diagnosis from nursing intervention and outcome] consists of the subcategory $<$ Consideration of nursing diagnosis based on the necessity of nursing intervention>, which stems from one nurse's comment that "There are probably a lot of people that feel uncertain what sort of nursing diagnosis should be done when they have determined what sort of care is appropriate, and they consider a nursing diagnosis from a nursing care perspective." In addition, this also includes the subcategory $<$ Consideration for the relationship between nursing diagnoses and outcome>, falling under situations where a nursing diagnosis is considered from the outcome of whether the nursing diagnosis is necessary for nursing intervention in response to ADL decline for the purpose of hospital discharge, or if the nursing diagnosis is in response to a required state of repose due to an illness.

[Patterned thinking relating to specific diagnoses] has several categories, including $<$ Nursing diagnosis linked with specific patient conditions $>$, such as one nurse who always gives a diagnosis of "Risk of Falls" for orthopedic surgery patients, $<$ Nursing diagnoses linked with past experiences $>$, as explained by a nurse that claimed she would worry about which nursing diagnosis should be referenced at first, but came to be able to determine which to use without hesitation if it was one that she was familiar with, and $<$ Nursing diagnoses linked with defining characteristics and related factors $>$, wherein one nurse explained that she gives a diagnosis of "Ineffective Protection" for patients that have decreased white blood cells or are receiving anticancer drug treatment.

[Planning a temporary nursing diagnosis and watching the progress] consists of the two subcategories $<$ Referencing nursing diagnoses while lacking information $>$ and $<$ Referencing nursing diagnoses when unsure $>$. Examples of the former include comments such as "Sometimes if I feel uncertain about a nursing diagnosis I'll use it to keep an eye on a patient for a week. I keep records and note the nursing diagnosis and date of assessment," and "There are many times where I go ahead with implementing a nursing diagnosis. If it's not a match I can always change it, and a change in condition allows me to give a diagnosis that is more in line with the symptoms." [Identifying multiple nursing diagnoses] consists of five subcategories. <Identification based on condition and restrictions $>$ refers to situations such as determining whether a diagnosis is "Risk for Aspiration" or "Impaired Swallowing" by knowing whether they are prohibited from eating or are undergoing training for swallowing.

$<$ Identification from priority of nursing intervention based on the patient's condition $>$ falls under times such as where "Fatigue" and "Impaired Comfort" are diagnosed as "Impaired Skin Integrity" depending on whether eliminating pain is a priority due to the patient's condition based on their progress with an increased dosage of narcotics, as well as times where uncertainty with diagnosis between "Risk for Falls" and "Toileting Self-Care Deficit" results in 
nursing diagnosis changing course after judging that the focus should be placed on self-care assistance in a patient's life. <Identification based on definition of nursing diagnoses $>$ includes cases such as when moving from "Acute Pain" to "Chronic Pain," where "Acute Pain" is chosen depending on how many days have passed. $<$ Identification by confirming the number of conforming defining characteristics and related factors $>$ includes determination by figuring out that the defining characteristics fall in line with a certain nursing diagnosis, and even more so by checking what related factors exist. Moreover, $<$ Comparison of defining characteristics and definitions for nursing diagnosis approximated from problems $>$ is from a case where a patient's problems were thought to indicate "Fatigue" and "Impaired Comfort," but the definitions and diagnostic indicators were verified once more despite an inclination to go with "Impaired Comfort."

[Integrating multiple nursing diagnoses] consists of the two subcategories <Integrating to reduce burden and confusion due to overlapping nursing intervention and evaluation $>$ and $<$ Integration to design high priority nursing diagnoses $>$. Examples of the former included comments such as "There are times where I consider giving a nursing diagnosis of 'Diarrhea' and then I find there's also 'Impaired Skin Integrity,' and it becomes too much of a load giving so many, so in the end I do a nursing intervention with just 'Ineffective Protection,", and "When providing mobility assistance for patients with 'Impaired transfer ability' and 'Risk for Falls,' the nursing intervention I provide is basically done for both nursing diagnoses, but since it can get confusing, I find myself wondering if just a single nursing diagnosis would be fine."

Subcategories for [Avoiding determining some nursing diagnoses] include <Avoiding determining impossible nursing diagnoses>, exemplified by the comment that "Since there isn't much information available for a patient's emergency hospitalization, there are times where I decide to hold off on determining a nursing diagnosis, watch their condition throughout the day, and plan a diagnosis in the day shift," and <Avoiding determining unfamiliar nursing diagnoses>, explained by one nurse as "There are many cases where patients exhibit anxiety, making it difficult to devise a plan for nursing diagnosis, so I end up just keeping an eye on their condition."

\section{Behavioral perspective of nurses in the process of determining a nursing diagnosis}

The three categories of [Confirming and reviewing with other staff when determining a diagnosis], [Solo confirmation and review to determine a nursing diagnosis], and [Taking action without confirming] were selected from the behavioral perspective of nursing in the process of determining a nursing diagnosis and consisted of a further 11 subcategories.

The category [Confirming and reviewing with other staff when determining a nursing diagnosis] includes subcategories such as $<$ Confirming and reviewing at conferences $>$, which stems from responses such as “I didn't study making decisions for nursing diagnoses when I was in school, and I don't have the confidence for self-study, so I review at conferences." In addition to this is $<$ Separate consultation with medical staff $>$, which comes from statements including "I don't worry about it alone and consult with staff that can take the lead or colleagues like committee members," "I consult a little with members on my shift and don't decide diagnoses by myself," and "I ask around for information, like asking doctors about treatment approaches."

[Confirming and reviewing with other staff to decide on a diagnosis] consists of the categories $<$ Referencing "NANDA International Nursing Diagnoses: Definitions and Classification" for confirmation>, $<$ Taking a cursory look at "NANDA International Nursing Diagnoses: Definitions and Classification" for confirmation $>$, $<$ Taking a cursory look at "NANDA International Nursing Diagnoses: Definitions and Classification" $>$, and $<$ Collecting information from patients and families>, which come from one nurse's habit of looking at the NANDA-I while also making a conscious effort to listen to what patients and their families want during hospitalization when giving a nursing diagnosis.

Other subcategories include <Referencing hospital training materials $>$, where case study conferences held within the hospital that also review cases from affiliated wards and commentary materials are utilized for reference. In addition to this is $<$ Researching the meaning of expressions for unfamiliar nursing diagnoses $>$, stemming from the comments, "If there are any expressions we see when referencing the NANDA-I book for diagnoses that we don't normally use, we first research that expression," and "Sometimes a definition isn't easy to understand, so we look it up in a dictionary for confirmation." Another subcategory was <Investigating reference materials>, wherein materials that were taught in university are drawn out for reference.

[Taking action without confirming] consists of the three subcategories $<$ Making specific treatment method entries freely instead of a nursing diagnosis $>$, <Inputting specific nursing intervention methods into integrated nursing diagnoses $>$, and $<$ Inputting nursing diagnoses directly without confirming with "NANDA International Nursing Diagnoses: Definitions and Classification">.

\section{Emotional perspective of nurses in the process of determining a nursing diagnosis}

The five categories of [Pressure to do a nursing diagnosis], [Sense of burden for task load that goes with nursing diagnosis], [Anxiety when determining a nursing diagnosis], [Negative emotions towards nursing diagnosis], and [Decline of negative emotions towards nursing diagnosis] were selected for the emotional perspective of nursing in the process of determining a nursing diagnosis, with a further 11 subcategories.

[Pressure to do a nursing diagnosis] includes the subcategories $<$ Sense of duty to determine a nursing diagnosis $>$ and $<$ Sense of responsibility that comes with making unfamiliar nursing diagnosis $>$, both stemming from situations where a diagnosis must be implemented.

[Sense of burden for task load that goes with nursing diagnosis] includes the subcategories $<$ Sense of burden for increase of record volume consequent to number of nursing diagnoses $>$, which comes from feeling obligated to build up one's nursing records due to the number of diagnoses, and $<$ Sense of burden for tasks consequent to the evaluation process $>$, which comes from the obligation to evaluate each diagnosis one by one once proposing them.

[Anxiety when determining a nursing diagnosis] consists of two subcategories. One is <Anxiety from deciding on a nursing diagnosis $>$, which is seen in comments including "There are times where I am implementing a diagnosis while wondering to myself if this is really the right one, which gives me anxiety," and "I get uneasy and wonder if this is really the proper diagnosis if it's a nursing diagnosis that I've never used before or have only occasionally used." The other subcategory is <Anxiety towards the number of nursing diagnoses $>$, which relates to the comment that "There's this dreaded feeling when making a nursing diagnosis where you wonder if you should be doing something else."

[Negative emotions towards nursing diagnosis] derives from subcategories such as $<$ Bitterness towards nursing diagnosis $>$, which, as one nurse described, "Even nurses who have been doing this for 10 years feel hesitant to suggest a nursing diagnosis. It's sort of like self-education in the sense that you learn by watching." Among other subcategories was $<$ Reluctance towards nursing diagnoses $>$.

[Decline of negative emotions towards nursing diagnosis] is made up of three subcategories. $<$ Reduced hesitancy thanks to hospital training system $>$ relates to one nurse's comment that "I didn't 
understand anything at first, but I was given paper patient records and the whole hospital ward worked on the diagnosis. During our conference time committee members explained things for me, which was a great help and dispelled how difficult I thought it would be, so that got rid of any bitterness I felt. That study meeting really saved me." $<$ Reduced sense of burden thanks to surrounding support and agreement $>$ derives from the comments, "If there is agreement among staff, it's not as worrisome even if there is some ambiguity with the nursing diagnosis," and "I can get help if I can't make a decision by myself, so I'm thankful for those around me." $<$ Reduced sense of burden thanks to routine and practice> stems from the remark that "All staff study and become familiarized with nursing diagnosis, so it's becoming established. I can use a nursing diagnosis with no problem for patients in my department."

\section{Needs for the use of nursing diagnoses}

Needs for the application of nursing diagnosis include the seven categories of [Needs for fostering understanding and learning of nursing diagnosis], [Needs for expanding the process of nursing diagnosis], [Needs for nursing diagnosis and nursing intervention for patients in specific situations], [Needs for the application of an electronic health record system], [Needs for nursing diagnosis in situations with insufficient patient information], [Needs for thinking ability in applying nursing diagnosis], and [Needs for sharing of information among staff], consisting of a further 22 subcategories.

[Needs for fostering understanding and learning of nursing diagnosis] includes five subcategories relating to viewpoints and methods to help understand nursing diagnoses. This includes $<$ Needs for fostering understanding of diagnoses, definitions, and defining characteristics $>$, based upon comments such as "If the definition and defining characteristics for a nursing diagnosis uses terms that are somewhat easy to understand or familiar, they're easy to use. If the nursing diagnosis is something I don't understand, I settle on taking a bit of time to look up terms since it would take too long to do research and I'm not getting any work done," as well as "I think it would be good if defining characteristics and definitions were a little easier to understand. The details of the wording for definitions differ somewhat from an actual clinical setting and don't really match." Comments such as "Our generation doesn't learn nursing diagnosis in school, and consideration of nursing diagnosis is important for actual patient cases," "In situations where you have usage examples of nursing diagnosis, or nursing diagnoses that you would not normally use, it would be best if they gave examples showing when specific nursing diagnoses should be used," and "It's difficult whether you have a nursing diagnosis or defining characteristics. If there was a manual that more easily explained in what situation or for what symptoms you would implement a certain nursing diagnosis, it would help for understanding details of nursing diagnoses that you can't put an image to" make up the subcategory of $<$ Needs for usage examples of case development and nursing diagnoses $>$. Other subcategories are $<$ Needs for understanding the foundation of identification of similar diagnoses $>$ and $<$ Needs for learning opportunities that match clinical settings $>$, and $<$ Needs for understanding the theory underlying nursing diagnoses $>$.

[Needs for nursing diagnosis and nursing intervention for patients in specific situations] contains three subcategories. $<$ Needs for nursing diagnosis and nursing intervention for terminal stage patients $>$ was seen in comments such as "There was a terminal stage patient with a bad prognosis and I wanted to understand how they were feeling, what they were thinking and offer my support. But I didn't know what nursing diagnosis I should be listening to those thoughts with, and in situations where they had numerous complaints such as 'Fatigue' or 'Impaired Comfort,' I felt anguished and conflicted, and didn't understand what nursing diagnosis I should have implemented the nursing intervention with" and "There are terminal patients that I do nursing interventions for, with a nursing diagnosis of Impaired Comfort and while they could have a prognosis of six months, they're still capable of handling every day activities by themselves and stay outside of the hospital. There are so many things to worry about at numerous stages such as intensity and intervals of pain that it makes me wonder if this is the correct nursing diagnosis." Other subcategories include $<$ Needs for nursing diagnosis and nursing intervention for patients requiring emotional support $>$ and $<$ Needs for nursing diagnosis and nursing intervention of families $>$.

[Needs for the use of an electronic health record system] consists of four subcategories. $<$ Needs for using registered nursing intervention entries in electronic health records $>$ is derived from the comment that "The nursing intervention part of the electronic health records has some things already filled in that you just go over, but it's all too obvious and I mostly end up putting in my own entries once I consider the uniqueness of my case," while $<$ Needs for characteristics of structure for electronic health records $>$ is seen in comments including one that "There is so much to see on an electronic health record, and I haven't clicked on everything, so there's a lot to go over, such as doctor records, medical records, nursing records, examination data, and fever charts." $<$ Needs for joint use between electronic health record systems and other media $>$ and $<$ Needs for individually different usage methods of electronic health records $>$ derive from comments including "We use both printed mediums and electronic health records, so it gets complicated having to look at and verify electronic records, critical paths, and daily worksheets."

[Needs for thinking ability in applying nursing diagnosis] includes the two subcategories <Needs in response to adverse effects of selecting diagnoses and nursing interventions on a computer $>$ and $<$ Adverse effects stemming from name of diagnosis used $>$, as seen in the response "I think the biggest problem with electronic health records is that your thinking ability declines. It feels like just checking off and implementing a nursing diagnosis without putting much thought into the nursing of an individual patient or asking yourself what the main problem is, so that leads me to believe thinking ability has decreased since their introduction."

\section{Discussion}

Issues with the process for determining a nursing diagnosis from a thought perspective, and support to improve them

It was demonstrated that a trait in the thought perspective in the general process of determining a nursing diagnosis is the tendency to focus on main symptoms, problems, nursing intervention, or outcome to give a candidate for nursing diagnosis that is then compared to the defining characteristics.

Another characteristic trend in the way nurses think was their patterned thinking for specific nursing diagnoses. Nurses who practice clinical nursing daily in particular will develop a pattern from their repeated experiences with similar situations of patient care, and there also seems to be an unwritten rule appearing within wards where a nursing diagnosis is made by combining these experiences with defining characteristics and related factors. Benner et al. explain that when grasping a patient's condition and providing support, experienced nurses provide pattern recognition, recognition of similarities, common understanding, proficient and practical knowledge, the ability to notice important points, and a rationale gained through experience [8]. This thinking pattern in the process of determining a nursing diagnosis is thought to be connected with their ability, cultivated through experience, to recognize similarities and perceive important points. Thus, we consider the increasing focus on intuitive nursing diagnosis during a nurse's experiences and find that it is an effective way of thinking in terms of making effective progress for nursing diagnoses. On the other hand, patterned thinking is uniform and not analytical, and thus can potentially lead to insufficient support for individual cases and insufficient review of the information of individual patients. Bearing these disadvantages in mind, however, it is essential that intuitive judgment continues to be applied. 
Another trend that has been demonstrated for clinical settings is the sense of burden that accompanies giving too many nursing diagnoses, as well as the tendency to integrate nursing diagnoses when nursing intervention overlaps. This integration also takes into consideration the importance of nursing intervention and the outcome that the patient requires. It is thought that the anxiety and sense of burden when making a nursing diagnosis is reduced once clinical nurses have an indicator or hint concerning the thought processes for this integration.

Issues with the decision-making process for nursing diagnosis from an emotional and thought perspective, and support to improve them

The results indicate that nurses experienced pressure to provide a nursing diagnosis. It was pointed out in a Korean study that some nurses complained that using standardized nursing languages, such as the NANDA-I, was a burden, as they do not have enough time. This study suggested it would take extra time to write nursing documentation if they do not have enough knowledge about SNLs [9]. Ofi and Sowunmi said that lack of time and knowledge, and the need for extensive writing are the major barriers against documentation [10]. Nurses felt there was a lack of education for nursing diagnoses and recognized its necessity, and also felt anxiety when making a diagnosis. This suggested that the knowledge of nursing diagnoses and time for documentation influenced feelings of being burdened.

For that reason, they did not make independent decisions for nursing diagnoses and regularly carried out multiple forms of verification through conferences and consultation. This emphasis on determining nursing diagnoses through confirmation and agreement with groups shows that the unwritten rules in all wards are easily influenced when reviewing nursing diagnoses in groups, and is assumed to also influence patterned thinking. While it was indicated that conferences are effective for a nurse's state of mind, further confirmation of their effect in terms of validity and accuracy will be required.

\section{Needs for the use of nursing diagnoses and support measures}

Nurses brought up the need for fostering understanding of nursing diagnosis terminology and called for explanation and discussion using specific examples. Clinical nurses recognize the issue with understanding defining characteristics and definitions and sought methods that convey terminology more easily.

"Needs for the use of an electronic health record system" also came up, which in turn suggests the need to provide support in utilizing the electronic health record system. Meißner and Schnepp point out that the quality of records can help improve the quality of care, and state that the time needed to complete electronic health records depends on effective use of IT [11].In addition, the two argues that in addition to the attitude of the nurses, ease of use and ability to use IT and equipment availability and technical functionality also affects the effective use of IT. As regards to the emotional perspective of the nurses, the sense of burden in completing and recording nursing diagnosis was mentioned. This suggests that there is a need to provide both user support and physical support, can help improve the quality of care, and state that the time needed to complete electronic health records depends on effective use of IT [11].In addition, the two argues that in addition to the attitude of the nurses, ease of use and ability to use IT and equipment availability and technical functionality also affects the effective use of IT. As regards to the emotional perspective of the nurses, the sense of burden in completing and recording nursing diagnosis was mentioned. This suggests that there is a need to provide both user support and physical support, et al. suggested the following four factors based on a systematic literature review: the nurse as a diagnostician, diagnostic education and resources, complexity of a patient's situation, and hospital policy and environment [12]. Considering previous study results such as above and the results of this research, we can argue that in order to make effective use of nursing diagnosis, it is crucial to clarify the policies within the nursing section of hospitals, and to provide educational support and environment for lack of learning. The NANDA-I explains the need for leaders in the nursing industry and nursing information specialists to work together to introduce an inspection system that can verify the accuracy of nursing diagnoses by using defining characteristics obtained from patient assessments and related/risk factors. Future cooperation with nursing information specialists will be needed to advance the application of nursing diagnosis.

\section{Limitations}

Since the participants of this study were nurses from a portion of one facility in Japan, the findings may have been influenced by the characteristics of said facility's department and the education background of its nurses. However, the facility is a mid-scale hospital that is unique for having around 500 beds and is thought to be representative of the characteristics of nurses working in Japan. Increasing the number of regions and facilities and further investigating whether similar situations exist will need to be attempted.

\section{Conclusions}

Ten categories were selected upon viewing the experiences of nurses in determining nursing diagnoses from a thought perspective. These categories are [Comparison with defining characteristics], [Confirmation of defining characteristics, related factors, and definition], [Searching for nursing diagnosis candidates from main symptoms and problems], [Considering nursing diagnosis from nursing intervention and outcome], [Patterned thinking relating to specific diagnoses], [Considering some temporary nursing diagnoses and monitoring progress], [Identification of multiple nursing diagnoses], [Comparison of some nursing diagnoses by picking potential diagnoses of defining characteristics or related factors, definition], [Integrating multiple nursing diagnoses], and [Avoiding determining some nursing diagnoses]. These categories revealed that there is no general nursing diagnosis process, and nurses will search for nursing diagnoses from main symptoms and nursing intervention or have a patterned thought process. From the behavioral and emotion perspectives, emphasis was placed on [Confirming and reviewing with other staff to determine a diagnosis] and [Solo confirmation and review to decide on a nursing diagnosis]. These were indicated to be driven by the following: [Pressure to do a nursing diagnosis], [Sense of burden for task load that goes with nursing diagnoses], [Anxiety when determining a nursing diagnosis], [Negative emotions towards nursing diagnoses], and [Decline of negative emotions towards nursing diagnoses].

\section{Conflict of Interest}

The authors declare that they have no competing interests.

\section{Acknowledgments}

We extend our thanks to all the participants in this study. This work was supported by JSPS KAKENHI Grant Number 15K11537. The authors declare no conflict of interest associated with this manuscript.

\section{References}

1. Herdman T, Kamitsuru S, (2014) From assessment to diagnosis. In: T Herdman \& S Kamitsuru (Eds.), NANDA International Nursing Diagnoses: Definitions and Classification 2015-2017 (10th ed.) p. 464. Wiley Blackwell, Oxford.

2. Hsiao CJ, Hing E (2012) Use and characteristics of electronic health record systems among office-based physician practices: United states, 2001-2012. NCHS Data Brief 1-8.

3. Japanese Association of Healthcare Information Systems Industry (2016) Report on situation of introduction of ordering and electronic health record systems in hospitals. 1-2. 
4. Shiraishi S, Fujiki M, Takemasa N, Hosako K, Yatabe K, et al. (2015) Seishin kangoryouiki ni okeru kangoshindandonyuu nikansuru zenkoku jittaichousa [National wide survey of introducing nursing diagnosis in psychiatric nursing units]. J Japan Soc Nurs Diagn 20: 4-13.

5. Hasegawa T, Ogasawara C, Katz EC (2007) Measuring diagnostic competency and the analysis of factors influencing competency using written case studies. Int J Nurs Know 18: 93-102.

6. Korthagen FAJ (1999) Linking reflection and technical competence: The logbook as an instrument in teacher education. E Euro J Teacher Edu 22: 191-207.

7. Graneheim UH, Lundman B (2004) Qualitative content analysis in nursing research: Concepts, procedures and measures to achieve trustworthiness. Nurs Edu Today 24: 105-112.

8. Benner P, Tanner C (1987) Clinical judgment: How expert nurses use intuition. Am J Nurs 87: 23-31.

9. Park H, Lee E (2015) Incorporating Standardized Nursing Languages In to an Electronic Nursing Documentation System in Korea: A Pilot Study. Int J Nurs Knowl 26: 35-42.

10. Ofi B, Sowunmi O (2012) Nursing documentation: Experience of the use of the nursing process model in selected hospitals in Ibadan, Oyo State, Nigeria. Int J Nurs Pract 18: 354-362.

11. Meißner A, Schnepp W (2014) Staff experiences within the implementation of computer-based nursing records in residential aged care facilities: a systematic review and synthesis of qualitative research. BMC Medical Informatics and Decision Making.

12. Paans W, Nieweg RM, van der Schans CP, Sermeus W (2011) What factors influence the prevalence and accuracy of nursing diagnoses documentation in clinical practice? A systematic literature review. J Clin Nurs 20: 2386-2403. 